\title{
Resíduos de calda sulfocálcica sobre a eficiência de acaricidas no controle de Brevipalpus phoenicis
}

\author{
Lime sulfur residue on acaricide efficiency in the control of Brevipalpus phoenicis
}

\section{Daniel Junior de Andrade $^{\mathrm{I} *}$ Fernando César Pattaro $^{\mathrm{I}}$ Carlos Amadeu Leite de Oliveira $^{\mathrm{I}}$}

\section{RESUMO}

\begin{abstract}
O objetivo do trabalho foi verificar a influência dos resíduos de calda sulfocálcica sobre a eficiência de acaricidas empregados no controle de Brevipalpus phoenicis. Inicialmente, o experimento foi instalado em um pomar de citros, em que foram selecionadas 10 plantas que continham frutos com verrugose. Realizou-se a aplicação de calda sulfocálcica (8g i.a. $L^{-1}$ de água) em cinco plantas e as outras cinco plantas permaneceram sem aplicação de produto fitossanitário. Protegeram-se, com copos de plástico transparente de $500 \mathrm{~mL}$, 64 frutos nas plantas tratadas com calda e 64 frutos nas plantas não-tratadas, totalizando 128 frutos protegidos. Decorridos 30 dias da aplicação, os frutos foram colhidos e levados para o laboratório. Estes frutos foram parcialmente parafinados, deixando-se em cada fruto uma arena de $2,5 \mathrm{~cm}$ de diâmetro com verrugose e sem parafina, delimitada com cola entomológica. Em seguida, procedeu-se à aplicação sobre os frutos em Torre de Potter dos seguintes acaricidas nas concentrações expressas em mg de ingrediente ativo por litro de água: propargite a $720 \mathrm{mg}$, óxido de fenbutatina a 400mg, cyhexatin a 250mg, azocyclotin a $250 \mathrm{mg}$, fenpyroximate a 50mg, dicofol a 960mg e dinocap a $738 \mathrm{mg} e$ a testemunha sem aplicação de acaricida. Após 1, 7 e 16 dias da aplicação, transferiram-se para cada fruto 10 ácaros $\boldsymbol{B}$. phoenicis para avaliar a mortalidade. Constatou-se que os resíduos de calda sulfocálcica não prejudicaram a eficiência dos acaricidas avaliados no controle de B. phoenicis.
\end{abstract}

Palavras-chave: leprose dos citros, controle químico, citricultura.

\section{ABSTRACT}

The objective was to assess the influence of lime sulfur residues on the acaricidal efficiency against Brevipalpus

\begin{abstract}
phoenicis mite. Initially, the experiment was conducted in a citrus orchard where 10 plants presenting scab fruits were selected. Secondly, the application of lime sulfur (8mg a.i. $L^{-1}$ of water) was performed in five plants, and the other five plants remained without application. Next lime sulfur application, 64 fruits in the plants with lime sulfur application were protected with plastic cups and others 64 fruits also were protected in the plants without lime sulfur application with plastic cups. After 30 days of field application, fruits were harvested and transported to the laboratory. These fruits were partially waxed, leaving an arena of approximately $2.5 \mathrm{~cm}$ in diameter with scab and without paraffin and entrapped with entomological glue. Afterwards, acaricides application were done following the concentration expressed in mg of active ingredient per liter of water: propargite (720mg), fenbutatin oxide (400mg), cyhexatin (250mg), azocyclotin (250mg), fenpyroximate $(50 \mathrm{mg})$, dicofol $(960 \mathrm{mg})$, dinocap (738mg) and control treatment by using the petri dish-Potter tower method. After 1, 7 and 16 days of application, ten B. phoenicis were transferred per fruit, in order to evaluate their mortality, 24 and 48 hours after the transferences. The lime sulfur residue did not affect the acaricides efficiency.
\end{abstract}

Key words: citrus leprosis, chemical control, citriculture.

\section{INTRODUÇÃO}

O ácaro da leprose dos citros Brevipalpus phoenicis (Geijskes, 1939) (Acari: Tenuipalpidae) é uma das principais pragas da citricultura por ser vetor do vírus CiLV (Citrus leprosis virus) causador da leprose dos citros, uma das doenças mais graves da citricultura no Brasil, devido principalmente aos gastos necessários

'Laboratório de Acarologia, Departamento de Fitossanidade, Faculdade de Ciências Agrárias e Veterinárias (FCAV), Universidade Estadual Paulista (UNESP), Via de acesso Prof. Paulo Donato Castellane, s/n, 14884-970, Jaboticabal, SP, Brasil. E-mail: danieldwv@yahoo.com.br.*Autor para correspondência. 
para o controle do ácaro vetor (RODRIGUES, et al., 2003; BASTIANEL et al., 2010).

O controle de B. phoenicis, com aplicações de acaricidas sintéticos, tem sido atualmente a principal tática de manejo da leprose dos citros e responsável por uma parcela significativa dos custos de produção (BASTIANEL et al., 2010; ANDRADE et al., 2010). Diante desse cenário, devido principalmente ao baixo valor pago pela caixa de laranja nos últimos anos, a calda sulfocálcica voltou a ser empregada na citricultura, já que constitui uma alternativa, além de reduzir o número de aplicações de agrotóxicos em cultivos convencionais, pode melhorar a produção em cultivos orgânicos (FERNANDES, 2001)

A calda sulfocálcica é um produto de uso frequente na agricultura orgânica, sendo empregada principalmente no controle dos ácaros B. phoenicis e Phyllocoptruta oleivora (Ashmead, 1879) e de algumas cochonilhas (PATTARO, 2003). Em citros orgânicos, a calda sulfocálcica é um dos poucos produtos químicos aceitos pelo Instituto Biodinâmico de Desenvolvimento Rural - IBD (PATTARO, 2003). É um sulfurado inorgânico que possui como princípio ativo o polissulfeto de cálcio, obtido pela dissolução de enxofre em pó e cal virgem ou hidratada. De acordo com ABBOTT (1945), a toxicidade da calda deve-se à liberação dos gases tóxicos sulfeto de hidrogênio $\left(\mathrm{H}_{2} \mathrm{~S}\right)$ e dióxido de enxofre $\left(\mathrm{SO}_{2}\right)$. A calda sulfocálcica possui ação fungicida, inseticida e acaricida, sendo utilizada também como fertilizante foliar (PRATES, 1999). Segundo BERTOLDO (2003), aplicações de calda sulfocálcica fornecem cálcio e enxofre ao metabolismo das plantas, que estimulam as reações de fotossíntese e induzem-nas à maior resistência às pragas.

Entretanto, em uma eventual necessidade de reaplicação, o citricultor comumente a faz utilizandose de um acaricida com maior período residual. Todavia, há a suspeita de que o carbonato de cálcio presente na calda sulfocálcica depositado sobre as plantas, possa interferir negativamente na eficiência de acaricidas específicos aplicados posteriormente.

Dessa forma, o presente trabalho teve por objetivo verificar a influência dos resíduos de calda sulfocálcica sobre a eficiência de acaricidas específicos no controle do ácaro da leprose B. phoenicis.

\section{MATERIAL E MÉTODOS}

O experimento foi realizado em duas etapas: a primeira a campo e a segunda em laboratório.
Inicialmente, o experimento foi instalado em 2002, em um pomar de citros da variedade Pera, sem aplicação de produtos fitossanitários há vários meses, localizado na área experimental da Faculdade de Ciências Agrárias e Veterinárias da Universidade Estadual Paulista (FCAV/ UNESP), Campus de Jaboticabal-SP.

Neste pomar, foram selecionadas 10 plantas que continham frutos com aproximadamente $5 \mathrm{~cm}$ de diâmetro e com sintomas de verrugose causados pelo fungo Elsinoe fawcetti, devido a B. phoenicis ter maior preferência por frutos com superfície irregular (ALBUQUERQUE et al., 1995). Após a seleção das plantas, realizou-se a aplicação de calda sulfocálcica em cinco plantas, na concentração de $8 \mathrm{~g}$ i.a. $\mathrm{L}^{-1}$ de água, até além do ponto de escorrimento, com o auxílio de um pulverizador manual costal $\mathrm{Jacto}^{\circledast}$, e as outras cinco plantas foram deixadas sem aplicação. A calda sulfocálcica utilizada foi a Super $\mathrm{S} 20^{\circledR}$, fabricada pela Fertibom - Catanduva-SP, com a concentração de 200g de enxofre $\mathrm{L}^{-1}$ de produto comercial.

De modo a evitar a ação das chuvas diretamente sobre os frutos, especialmente naqueles tratados com calda sulfocálcica, protegeram-se, com copos de plástico transparente de $500 \mathrm{~mL}, 64$ frutos nas plantas tratadas com calda e 64 frutos nas plantas não-tratadas, totalizando 128 frutos protegidos. Durante a condução do experimento em campo, os dados meteorológicos foram registrados pela Estação Agroclimatológica da FCAV/UNESP, Campus de Jaboticabal-SP.

Decorridos 30 dias da aplicação no campo, os frutos com e sem resíduo de calda, com e sem proteção com os copos de plástico, foram colhidos e levados para o Laboratório de Acarologia da FCAV/ UNESP e dispostos em bandejas plásticas. No laboratório, os frutos foram parcialmente parafinados, deixando-se em cada fruto uma arena de $2,5 \mathrm{~cm}$ de diâmetro com verrugose e sem parafina, delimitada com uma barreira de cola entomológica (Tree Tanglefoot ${ }^{\circledR}$ ).

Foram estabelecidos vinte e quatro tratamentos, com oito repetições, em delineamento inteiramente casualizado, sendo que cada unidade experimental foi constituída por um fruto (Tabela 1). As aplicações dos acaricidas sobre os frutos foram realizadas em Torre de Potter, calibrada a 5lbf $\mathrm{pol}^{-2}$, empregando-se $2 \mathrm{~mL}$ de calda por fruto, o que corresponde a aproximadamente 16 a 20 litros por planta de porte médio (MATUO \& NAKAMURA, 1989).

Após a aplicação dos acaricidas, com auxílio de um pincel de apenas um pelo, foram transferidas 
Tabela 1 - Relação dos tratamentos estabelecidos no experimento de avaliação do efeito dos resíduos de calda sulfocálcica sobre a eficiência de acaricidas no controle do ácaro Brevipalpus phoenicis. Jaboticabal-SP, 2002.

\begin{tabular}{|c|c|c|c|c|}
\hline \multicolumn{2}{|c|}{--Produtos utilizados-- } & \multirow{2}{*}{$\begin{array}{c}\text { Concentração } \\
\text { mg de i.a } L^{-1} \text { de água }\end{array}$} & \multicolumn{2}{|c|}{ - } \\
\hline Nome comercial & Nomecomum & & $\begin{array}{l}\text { Com }(\mathrm{C}) \text { ou sem }(\mathrm{S}) \\
\text { resíduo de calda } \\
\text { sulfocálcica }(\mathrm{R})\end{array}$ & $\begin{array}{l}\text { Com }(\mathrm{C}) \text { ou sem }(\mathrm{S}) \\
\text { proteção com copo } \\
\text { plástico }(\mathrm{P})\end{array}$ \\
\hline 1. Omite $720 \mathrm{BR}$ & Propargite & 720 & $\mathrm{CR}$ & $\mathrm{CP}$ \\
\hline 2. Omite $720 \mathrm{BR}$ & Propargite & 720 & $\mathrm{CR}$ & SP \\
\hline 3. Omite $720 \mathrm{BR}$ & Propargite & 720 & SR & $\mathrm{CP}$ \\
\hline 4. Torque $500 \mathrm{SC}$ & Fenbutatina $^{1}$ & 400 & $\mathrm{CR}$ & $\mathrm{CP}$ \\
\hline 5. Torque $500 \mathrm{SC}$ & Fenbutatina $^{1}$ & 400 & $\mathrm{CR}$ & SP \\
\hline 6. Torque $500 \mathrm{SC}$ & Fenbutatina $^{1}$ & 400 & SR & $\mathrm{CP}$ \\
\hline 7. Sipcatin $500 \mathrm{SC}$ & Cyhexatin & 250 & $\mathrm{CR}$ & $\mathrm{CP}$ \\
\hline 8. Sipcatin $500 \mathrm{SC}$ & Cyhexatin & 250 & $\mathrm{CR}$ & SP \\
\hline 9. Sipcatin $500 \mathrm{SC}$ & Cyhexatin & 250 & SR & $\mathrm{CP}$ \\
\hline 10. Caligur 500SC & Azocyclotin & 250 & $\mathrm{CR}$ & $\mathrm{CP}$ \\
\hline 11. Caligur $500 \mathrm{SC}$ & Azocyclotin & 250 & $\mathrm{CR}$ & SP \\
\hline 12. Caligur $500 \mathrm{SC}$ & Azocyclotin & 250 & SR & $\mathrm{CP}$ \\
\hline 13. Ortus $50 \mathrm{SC}$ & Fenpyroximate & 50 & $\mathrm{CR}$ & $\mathrm{CP}$ \\
\hline 14. Ortus $50 \mathrm{SC}$ & Fenpyroximate & 50 & CR & SP \\
\hline 15. Ortus $50 \mathrm{SC}$ & Fenpyroximate & 50 & SR & $\mathrm{CP}$ \\
\hline 16. Kelthane $480 \mathrm{CE}$ & Dicofol & 960 & $\mathrm{CR}$ & $\mathrm{CP}$ \\
\hline 17. Kelthane $480 \mathrm{CE}$ & Dicofol & 960 & $\mathrm{CR}$ & SP \\
\hline 18. Kelthane $480 \mathrm{CE}$ & Dicofol & 960 & SR & $\mathrm{CP}$ \\
\hline 19. Karathane $369 \mathrm{CE}$ & Dinocap & 738 & $\mathrm{CR}$ & $\mathrm{CP}$ \\
\hline 20. Karathane $369 \mathrm{CE}$ & Dinocap & 738 & CR & SP \\
\hline 21. Karathane $369 \mathrm{CE}$ & Dinocap & 738 & SR & $\mathrm{CP}$ \\
\hline 22. Testemunha & - & - & CR & $\mathrm{CP}$ \\
\hline 23. Testemunha & - & - & $\mathrm{CR}$ & SP \\
\hline 24. Testemunha & - & - & SR & $\mathrm{CP}$ \\
\hline
\end{tabular}

${ }^{1}$ Óxido de Fenbutatina

para cada fruto 10 fêmeas adultas de $\boldsymbol{B}$. phoenicis, após, 1,7 e 16 dias da aplicação dos acaricidas. Os ácaros da leprose utilizados no experimento foram obtidos de uma criação-estoque. Foram realizadas avaliações 24 e 48 horas após cada transferência de B. phoenicis para os frutos, quantificando-se o número de ácaros mortos, vivos e retidos na barreira adesiva de cola entomológica.

Os dados obtidos referentes ao número de ácaros vivos e mortos foram transformados em porcentagem de sobrevivência para cálculo da eficiência dos tratamentos (mortalidade corrigida), utilizando-se a fórmula de Schneider - Orelli (NAKANO et al., 1981). Os dados de eficiência foram transformados em arco seno $\sqrt{P / 100}$, com o objetivo de normalizar os dados a serem analisados pelo teste $\mathrm{F}$, e as médias foram comparadas pelo teste de Tukey, a $5 \%$ de probabilidade.

\section{RESULTADOS E DISCUSSÃO}

Durante o período em que os frutos permaneceram no campo, os dados meteorológicos registrados pela Estação Agroclimatológica foram: temperatura média de $24,5^{\circ} \mathrm{C}$, umidade relativa do ar de $64,9 \%$ e precipitação total de $25,1 \mathrm{~mm}$.

Na tabela 2, são apresentados os resultados referentes à porcentagem de eficiência para cada acaricida nos diferentes tratamentos. Pode-se observar que não houve diferenças estatísticas entre os acaricidas, independentemente dos frutos, conterem ou não resíduo de calda sulfocálcica, ou estarem protegidos ou não com copos de plástico.

Embora a eficiência dos acaricidas propargite e fenpyroximate não tenha diferido estatisticamente entre os tratamentos com e sem resíduo de calda sulfocálcica, verificou-se que a 
Tabela 2 - Médias de porcentagem de eficiência (\%) nas transferências realizadas a 1, 7 e 16 dias da aplicação dos acaricidas, com (CR) e sem resíduo (SR) de calda sulfocálcica e com proteção (CP) e sem proteção (SP) com copo de plástico. Jaboticabal-SP, 2002.

\begin{tabular}{|c|c|c|c|c|c|c|}
\hline \multirow[t]{2}{*}{ Acaricidas } & ------------- & P------------- & ------------. & ------------ & ------------ & P-----------. \\
\hline & 24 horas & 48 horas & 24 horas & 48 horas & 24 horas & 48 horas \\
\hline Propargite & $70,3 \mathrm{aA}$ & $92,3 \mathrm{aA}$ & $75,6 \mathrm{aA}$ & $94,2 \mathrm{aA}$ & $73,3 \mathrm{aA}$ & $92,3 \mathrm{aA}$ \\
\hline Óxido de fenbutatina & $82,3 \mathrm{aA}$ & $95,7 \mathrm{aA}$ & $83,3 \mathrm{aA}$ & $98,1 \mathrm{aA}$ & $75,4 \mathrm{aA}$ & $96,6 \mathrm{aA}$ \\
\hline Cyhexatin & $99,2 \mathrm{aA}$ & $100,0 \mathrm{aA}$ & $98,7 \mathrm{aA}$ & $100,0 \mathrm{aA}$ & $94,6 \mathrm{aA}$ & $100,0 \mathrm{aA}$ \\
\hline Azocyclotin & $100,0 \mathrm{aA}$ & $100,0 \mathrm{aA}$ & $98,7 \mathrm{aA}$ & $100,0 \mathrm{aA}$ & $99,2 \mathrm{aA}$ & $100,0 \mathrm{aA}$ \\
\hline Fenpyroximate & $71,2 \mathrm{aA}$ & $93,6 \mathrm{aA}$ & $62,8 \mathrm{aA}$ & 84,1 aA & $61,4 \mathrm{aA}$ & $78,7 \mathrm{aA}$ \\
\hline Dicofol & $100,0 \mathrm{aA}$ & $100,0 \mathrm{aA}$ & $99,5 \mathrm{aA}$ & $100,0 \mathrm{aA}$ & $96,7 \mathrm{aA}$ & $100,0 \mathrm{aA}$ \\
\hline Dinocap & $99,2 \mathrm{aA}$ & $100,0 \mathrm{aA}$ & $98,3 \mathrm{aA}$ & $100,0 \mathrm{aA}$ & $97,9 \mathrm{aA}$ & $100,0 \mathrm{aA}$ \\
\hline Testemunha & $0,0 \mathrm{bA}$ & $0,0 \mathrm{bA}$ & $0,0 \mathrm{bA}$ & $0,0 \mathrm{bA}$ & $0,0 \mathrm{bA}$ & $0,0 \mathrm{bA}$ \\
\hline
\end{tabular}

${ }^{1}$ Médias seguidas pela mesma letra minúscula na coluna e maiúscula na linha não diferem significativamente, pelo teste de Tukey, a $5 \%$ de probabilidade.

eficiência desses acaricidas foi superior quando se utilizaram frutos com resíduo de calda sulfocálcica em comparação à aplicação de propargite e fenpyroximate sobre frutos sem resíduos de calda sulfocálcica. Provavelmente, os resíduos da calda sulfocálcica presentes sobre os frutos ainda apresentavam ação residual sobre os ácaros após 30 dias da aplicação no campo e contribuíram para o aumento da eficiência dos acaricidas propargite e fenpyroximate. Esses resultados concordaram com ALVES et al. (1999), embora a ação alcançada sobre B. phoenicis, no presente trabalho, tenha sido inferior.

Entretanto, para o acaricida fenpyroximate, as chuvas ocorridas podem ter sido responsáveis pela eliminação dos resíduos de calda sulfocálcica presentes nos frutos, explicando a menor eficiência de controle obtida em frutos com carbonato de cálcio e protegidos com copos de plástico, quando comparados àqueles frutos com carbonato de cálcio e não protegidos com copos de plástico. Todavia, de modo geral, os acaricidas avaliados ocasionaram efeitos semelhantes sobre $\boldsymbol{B}$. phoenicis quando aplicados em frutos tratados ou não com calda sulfocálcica, assim como em frutos protegidos ou não com copos de plástico.

Verificou-se que os acaricidas aplicados sobre os frutos que continham os resíduos da calda sulfocálcica e protegidos com copo plástico, nas avaliações realizadas 48 horas após as transferências, apresentaram, de maneira geral, maior porcentagem de eficiência. Contudo, conforme os resultados obtidos por ALVES et al. (2000), esperava-se, no presente trabalho, que os frutos com os resíduo de calda sulfocálcica e protegidos com copos de plástico proporcionassem maior controle de B. phoenicis, oque não foi constatado.

Após 24 horas das transferências, nos tratamentos com resíduo de calda sulfocálcica e protegidos com copos de plástico, observou-se que os acaricidas cyhexatin, azocyclotin, dicofol, dinocap apresentaram as maiores porcentagens de eficiência. Os resultados obtidos nos tratamentos com resíduo de calda e sem proteção, assim como nos tratamentos sem resíduo e com proteção, foram semelhantes para todos os acaricidas; entretanto, a eficiência, de maneira geral, foi superior nos tratamentos com resíduo de calda e com proteção, evidenciado um possível efeito sinérgico entre a calda sulfocálcica e os acaricidas avaliados.

Nas avaliações realizadas 48 horas após as transferências, constatou-se que ocorreu acréscimo significativo da porcentagem de eficiência em todos os tratamentos, indicando que o tempo de exposição do ácaro sobre os resíduos dos produtos aumentou consideravelmente a mortalidade dos ácaros. Assim como ocorreu quando foram aplicados sobre os frutos com resíduo de calda sulfocálcica, protegidos ou não, os acaricidas cyhexatin, azocyclotin, dicofol e dinocap também apresentaram o maior efeito sobre o ácaro $\boldsymbol{B}$. phoenicis, quando aplicados sobre frutos sem carbonato de cálcio, até 48 horas após as transferências, juntamente com óxido de fenbutatina. Nessas avaliações, o acaricida fenpyroximate foi o que 
proporcionou a menor mortalidade de ácaros $\boldsymbol{B}$. phoenicis.

É importante ressaltar que a média de ácaros vivos da testemunha, com aplicação somente de calda sulfocálcica no campo, observada após as transferências realizadas 1, 7 e 16 dias após a aplicação da calda evidenciou baixa ação residual. Esses resultados discordaram de OLIVEIRA et al. (2002), que verificaram que a calda sulfocálcica apresentou elevada eficiência residual sobre o ácaro da ferrugem $\boldsymbol{P}$. oleivora, entretanto PATTARO (2003) observou que a calda sulfocálcica, em doses de até $4 \mathrm{~L} 100 \mathrm{~L}^{-1}$ de água, apresenta baixa ação residual sobre adultos de $\boldsymbol{B}$. phoenicis, bem como baixa ação ovicida.

De maneira geral, verificou-se que os acaricidas aplicados sobre frutos que apresentavam resíduo de calda sulfocálcica, independentemente de estarem protegidos das chuvas, apresentaram porcentagens de eficiência superior aos acaricidas aplicados em frutos sem carbonato de cálcio (Tabela 3). Quanto ao período residual dos acaricidas, observouse, por meio das médias de porcentagem de eficiência apresentadas na tabela 3, que o residual dos acaricidas

Tabela 3 - Médias de porcentagem de eficiência (\%), 24 e 48 horas após as transferências, presentes nos frutos com e sem resíduo, com e sem proteção, e nas transferências realizadas a 1,7 e 16 dias da aplicação dos acaricidas. Jaboticabal-SP, 2002.

\begin{tabular}{|c|c|c|}
\hline \multirow{2}{*}{ Condição dos frutos no campo } & \multicolumn{2}{|c|}{$\begin{array}{l}\text { Horas após a } \\
\text { transferência }{ }^{1}\end{array}$} \\
\hline & 24 & 48 \\
\hline Frutos com resíduo e com proteção & 88,9 a & $97,4 \mathrm{a}$ \\
\hline Frutos com resíduo e sem proteção & $88,2 \mathrm{a}$ & 96,6 a \\
\hline Frutos sem resíduo e com proteção & 85,5 a & $95,4 \mathrm{a}$ \\
\hline TESTE F & $0,2^{\mathrm{ns}}$ & $0,1^{\mathrm{ns}}$ \\
\hline $\mathrm{CV}(\%)^{2}$ & 19,1 & 11,3 \\
\hline $\mathrm{EP}^{3}$ & 5,3 & 3,5 \\
\hline \multirow[t]{2}{*}{ Dias após as transferências } & \multicolumn{2}{|c|}{$\begin{array}{l}\text { Horas após a } \\
\text { transferência }{ }^{1}\end{array}$} \\
\hline & 24 & 48 \\
\hline 1 & 96,2 a & $98,9 \mathrm{a}$ \\
\hline 7 & $93,0 \mathrm{ab}$ & $99,6 \mathrm{a}$ \\
\hline 16 & $73,2 \mathrm{~b}$ & $90,9 \mathrm{~b}$ \\
\hline TESTE F & $5,4 * *$ & $7,0 * *$ \\
\hline $\mathrm{CV}(\%)^{2}$ & 22,3 & 11,1 \\
\hline $\mathrm{EP}^{3}$ & 3,7 & 2,1 \\
\hline
\end{tabular}

ns - não significativo e (**) significativo a $1 \%$ de probabilidade.

${ }^{1}$ Médias seguidas pela mesma letra na coluna não diferem significativamente pelo teste de Tukey, a $5 \%$ de probabilidade.

${ }^{2}$ Coeficiente de Variação;

${ }^{3}$ Erro padrão da média. decresce à medida que o tempo após a aplicação aumenta, diferindo entre si após cada transferência. A transferência dos ácaros realizada aos 16 dias após a aplicação foi a que proporcionou aos acaricidas as menores eficiências e, na avaliação realizada 48 horas após a transferência, observou-se que a eficiência média diferiu estatisticamente das médias obtidas pelas transferências realizadas a 1 e a 7 dias após a aplicação.

Verificou-se que os acaricidas propargite, óxido de fenbutatina e fenpyroximate, na transferência realizada aos 16 dias após a aplicação, independentemente dos frutos utilizados, apresentaram percentuais de eficiência inferior quando os ácaros foram transferidos 1 e 7 dias após a aplicação dos acaricidas (Tabela 4). Com isso, pode-se inferir que esses acaricidas apresentam menor efeito residual sobre B. phoenicis em relação aos demais acaricidas avaliados.

Com base nos resultados obtidos, constatou-se que a ação residual dos acaricidas avaliados não sofreu influência negativa da aplicação de calda sulfocálcica realizada anteriormente sobre os frutos. Nos tratamentos nos quais se verificou uma possível interferência, essa se mostrou positiva, constituindo o resíduo da calda sulfocálcica, principalmente o carbonato de cálcio, como um possível fator aditivo na mortalidade de B. phoenicis .

Quanto às testemunhas, constatou-se que não apresentaram diferença entre si, indicando resultados idênticos tanto para frutos com e sem resíduo, protegidos ou não. Disso infere-se que o resíduo de calda sulfocálcica, na dosagem de $4 \mathrm{~L}$ p.c. $100 \mathrm{~L}^{-1}$, após 30 dias da aplicação, não proporcionou mortalidade expressiva do ácaro B. phoenicis. Contudo, em épocas do ano em que haja reinfestação de $\boldsymbol{B}$. phoenicis, é possível a aplicação dos acaricidas avaliados após 30 dias da aplicação da calda sulfocálcica na dose de $4 \mathrm{~L} 100 \mathrm{~L}^{-1}$ de água. Todavia, a recomendação da calda sulfocálcica para controle de B. phoenicis deve ser sempre criteriosa e associada a outras estratégias de manejo, com o intuito de aumentar o período de controle, bem como evitar a seleção de populações de ácaros resistentes (PATTARO, 2003; CASARIN, 2010).

\section{CONCLUSÃO}

Os resíduos de calda sulfocálcica sobre frutos de laranja não interfere na eficiência dos acaricidas propargite, óxido de fenbutatina, cyhexatin, azocyclotin, fenpyroximate, dicofol e dinocap sobre a mortalidade do ácaro B. phoenicis.

Ciência Rural, v.41, n.10, out, 2011. 
Tabela 4 - Eficiência (\%) de diferentes acaricidas sobre B. phoenicis, em laboratório, 1, 7 e 16 dias após a aplicação (DAA) sobre frutos com (CR) e sem resíduo (SR) de calda sulfocálcica, com (CP) e sem proteção (SP), avaliados 24 e 48 horas após as transferências dos ácaros. Jaboticabal-SP, 2002.

\begin{tabular}{|c|c|c|c|c|c|c|c|}
\hline \multicolumn{2}{|c|}{ Descrição dos tratamentos } & \multicolumn{2}{|c|}{ - } & \multicolumn{2}{|c|}{ 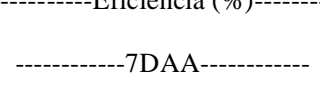 } & \multicolumn{2}{|c|}{----------16DAA--------- } \\
\hline Produtos & Frutos & $24 h^{2}$ & $48 \mathrm{~h}$ & $24 \mathrm{~h}$ & $48 \mathrm{~h}$ & $24 \mathrm{~h}$ & $48 \mathrm{~h}$ \\
\hline 1. Propargite & $\mathrm{CR}, \mathrm{CP}$ & $93,1 \mathrm{abc}^{3}$ & $94,5 \mathrm{a}$ & 94,5 abcd & $98,7 \mathrm{a}$ & $10,6 \mathrm{bcd}$ & $80,9 \mathrm{bc}$ \\
\hline 2. Propargite & $\mathrm{CR}, \mathrm{SP}$ & $100,0 \mathrm{a}$ & $100,0 \mathrm{a}$ & $95,8 \mathrm{ab}$ & $100,0 \mathrm{a}$ & $9,8 \mathrm{bcd}$ & $79,0 \mathrm{bc}$ \\
\hline 3. Propargite & $\mathrm{SR}, \mathrm{CP}$ & $98,7 \mathrm{ab}$ & $100,0 \mathrm{a}$ & $92,3 \mathrm{abcd}$ & $100,0 \mathrm{a}$ & 13,9 cde & $68,5 \mathrm{~cd}$ \\
\hline 4. Fenbutatina ${ }^{6}$ & $\mathrm{CR}, \mathrm{CP}$ & $93,0 \mathrm{abc}$ & $95,5 \mathrm{a}$ & $86,1 \mathrm{bcd}$ & 98,6 a & $57,7 \mathrm{~b}$ & $89,8 \mathrm{abc}$ \\
\hline 5. Fenbutatina ${ }^{6}$ & $\mathrm{CR}, \mathrm{SP}$ & $95,0 \mathrm{abc}$ & $100,0 \mathrm{a}$ & $87,8 \mathrm{bcd}$ & $100,0 \mathrm{a}$ & $50,6 \mathrm{~b}$ & $91,4 \mathrm{ab}$ \\
\hline 6. Fenbutatina ${ }^{6}$ & $\mathrm{SR}, \mathrm{CP}$ & $92,3 \mathrm{abc}$ & $100,0 \mathrm{a}$ & $80,3 \mathrm{~cd}$ & $100,0 \mathrm{a}$ & $27,8 \mathrm{bc}$ & $84,9 \mathrm{abc}$ \\
\hline 7. Cyhexatin & $\mathrm{CR}, \mathrm{CP}$ & $100,0 \mathrm{a}$ & $100,0 \mathrm{a}$ & $97,5 \mathrm{ab}$ & $100,0 \mathrm{a}$ & $100,0 \mathrm{a}$ & $100,0 \mathrm{a}$ \\
\hline 8. Cyhexatin & $\mathrm{CR}, \mathrm{SP}$ & $100,0 \mathrm{a}$ & $100,0 \mathrm{a}$ & $96,2 \mathrm{ab}$ & $100,0 \mathrm{a}$ & $98,5 \mathrm{a}$ & $100,0 \mathrm{a}$ \\
\hline 9. Cyhexatin & $\mathrm{SR}, \mathrm{CP}$ & $100,0 \mathrm{a}$ & $100,0 \mathrm{a}$ & $88,5 \mathrm{bcd}$ & $100,0 \mathrm{a}$ & $100,0 \mathrm{a}$ & $100,0 \mathrm{a}$ \\
\hline 10. Azocyclotin & $\mathrm{CR}, \mathrm{CP}$ & $100,0 \mathrm{a}$ & $100,0 \mathrm{a}$ & $100,0 \mathrm{a}$ & $100,0 \mathrm{a}$ & $100,0 \mathrm{a}$ & $100,0 \mathrm{a}$ \\
\hline 11. Azocyclotin & $\mathrm{CR}, \mathrm{SP}$ & $100,0 \mathrm{a}$ & $100,0 \mathrm{a}$ & $96,2 \mathrm{ab}$ & $100,0 \mathrm{a}$ & $100,0 \mathrm{a}$ & $100,0 \mathrm{a}$ \\
\hline 12. Azocyclotin & $\mathrm{SR}, \mathrm{CP}$ & $100,0 \mathrm{a}$ & $100,0 \mathrm{a}$ & $95,0 \mathrm{ab}$ & $100,0 \mathrm{a}$ & $100,0 \mathrm{a}$ & $100,0 \mathrm{a}$ \\
\hline 13. Fenpyroximate & $\mathrm{CR}, \mathrm{CP}$ & $79,7 \mathrm{bc}$ & $100,0 \mathrm{a}$ & $85,5 \mathrm{bcd}$ & $100,0 \mathrm{a}$ & $44,7 \mathrm{~b}$ & $81,9 \mathrm{abc}$ \\
\hline 14. Fenpyroximate & $\mathrm{CR}, \mathrm{SP}$ & $87,5 \mathrm{abc}$ & $98,7 \mathrm{a}$ & $87,5 \mathrm{abc}$ & $100,0 \mathrm{a}$ & 9,3 ef & $52,5 \mathrm{~d}$ \\
\hline 15. Fenpyroximate & $\mathrm{SR}, \mathrm{CP}$ & $76,0 \mathrm{c}$ & 88,5 a & $77,3 \mathrm{~d}$ & $93,4 \mathrm{a}$ & $15,3 \mathrm{de}$ & $49,3 \mathrm{~d}$ \\
\hline 16. Dicofol & $\mathrm{CR}, \mathrm{CP}$ & $100,0 \mathrm{a}$ & $98,7 \mathrm{a}$ & $100,0 \mathrm{a}$ & $100,0 \mathrm{a}$ & $100,0 \mathrm{a}$ & $100,0 \mathrm{a}$ \\
\hline 17. Dicofol & $\mathrm{CR}, \mathrm{SP}$ & $100,0 \mathrm{a}$ & $100,0 \mathrm{a}$ & $100,0 \mathrm{a}$ & $100,0 \mathrm{a}$ & $98,6 \mathrm{a}$ & $100,0 \mathrm{a}$ \\
\hline 18. Dicofol & $\mathrm{SR}, \mathrm{CP}$ & $100,0 \mathrm{a}$ & $100,0 \mathrm{a}$ & 96,3 a & $100,0 \mathrm{a}$ & 86,9 a & $98,3 \mathrm{a}$ \\
\hline 19. Dinocap & $\mathrm{CR}, \mathrm{CP}$ & $100,0 \mathrm{a}$ & $100,0 \mathrm{a}$ & $97,5 \mathrm{ab}$ & $100,0 \mathrm{a}$ & $100,0 \mathrm{a}$ & $100,0 \mathrm{a}$ \\
\hline 20. Dinocap & $\mathrm{CR}, \mathrm{SP}$ & $100,0 \mathrm{a}$ & $100,0 \mathrm{a}$ & $96,2 \mathrm{ab}$ & $100,0 \mathrm{a}$ & $100,0 \mathrm{a}$ & $100,0 \mathrm{a}$ \\
\hline 21. Dinocap & $\mathrm{SR}, \mathrm{CP}$ & $100,0 \mathrm{a}$ & $100,0 \mathrm{a}$ & $100,0 \mathrm{a}$ & $100,0 \mathrm{a}$ & $100,0 \mathrm{a}$ & $100,0 \mathrm{a}$ \\
\hline 22. Testemunha & $\mathrm{CR}, \mathrm{CP}$ & $0,0 \mathrm{~d}$ & $0,0 \mathrm{~b}$ & $0,0 \mathrm{e}$ & $0,0 \mathrm{~b}$ & $0,0 \mathrm{f}$ & $0,0 \mathrm{e}$ \\
\hline 23. Testemunha & $\mathrm{CR}, \mathrm{SP}$ & $0,0 \mathrm{~d}$ & $0,0 \mathrm{~b}$ & $0,0 \mathrm{e}$ & $0,0 \mathrm{~b}$ & $0,0 \mathrm{f}$ & $0,0 \mathrm{e}$ \\
\hline 24. Testemunha & $\mathrm{SR}, \mathrm{CP}$ & $0,0 \mathrm{~d}$ & $0,0 \mathrm{~b}$ & $0,0 \mathrm{e}$ & $0,0 \mathrm{~b}$ & $0,0 \mathrm{f}$ & $0,0 \mathrm{e}$ \\
\hline TESTE F & & $82,7 * *$ & $101,1 * *$ & $84,4^{* *}$ & $137,6^{* *}$ & $83,3 * *$ & $74,4^{* *}$ \\
\hline $\mathrm{CV}(\%)^{4}$ & & 12,2 & 10,9 & 12,4 & 9,0 & 17,6 & 14,0 \\
\hline $\mathrm{EP}^{5}$ & & 3,2 & 3,0 & 3,1 & 2,6 & 3,7 & 3,5 \\
\hline
\end{tabular}

ns - não significativo e $(* *)$ significativo a $1 \%$ de probabilidade.

${ }^{1}$ Dias após aplicação.

${ }^{2}$ Horas após a transferência de B. phoenicis para os frutos. As porcentagens de redução da população de B. phoenicis foram calculadas pela fórmula de Schneider-Orelli.

${ }^{3}$ Médias seguidas pela mesma letra na coluna não diferem significativamente pelo teste de Tukey, a 5\% de probabilidade. Dados originais transformados em arco seno $\sqrt{P / 100}$.

${ }^{4}$ Coeficiente de Variação (\%).

${ }^{5}$ Erro padrão da média.

${ }^{6}$ Óxido de Fenbutatina.

\section{REFERÊNCIAS}

ABBOTT, C.E. The toxic gases of lime-sulfur. Journal of Economic Entomology, Lanham, v.38, n.5, p.618-620, 1945.

ALBUQUERQUE, F.A. et al. Comportamento do ácaro $\boldsymbol{B}$. phoenicis (Geijskes, 1939) (Acari: Tenuipalpidae) em frutos de citros. In: OLIVEIRA, C.A.L. de; DONADIO, L.C. Leprose dos citros. Jaboticabal: FUNEP, 1995. p.77-90.

ALVES, E.B. et al. Resistência cruzada entre o dicofol e outros acaricidas em Brevipalpus phoenicis (Geijskes) (Acari: Tenuipalpidae). Anais da Sociedade Entomológica do
Brasil, Londrina, v.29, n.4, p.765-771, 2000. Disponível em: <http://www.scielo.br/pdf/aseb/v29n4/v29n4a17.pdf>. Acesso em: 10 jan. 2011. doi: 10.1590/S0301-80592000000400017.

ALVES, S.B. et al. Eficiência da calda sulfocálcica no controle do ácaro da leprose. In: AMBROSANO, E. Agricultura ecológica. Simpósio de agricultura ecológica. Encontro de agricultura orgânica. Guaiba: Agropecuária, 1999. p.395-398.

ANDRADE, D.J. et al. Acaricidas utilizados na citricultura convencional e orgânica: manejo da leprose e populações de ácaros fitoseídeos. Revista Brasileira de Fruticultura, Jaboticabal, v.32, p.1028-1037, 2010. Disponível em: <http:/ 
/www.scielo.br/pdf/rbf/2011 nahead/aop15210.pdf>. Acesso em: 28 jan. 2011. doi: 10.1590/S0100-29452011005000013.

BASTIANEL, M. et al. Citrus leprosis: centennial of an unusual mite virus pathosystem. Plant Disease, Saint Paul, v.94, p.284-292, 2010. Disponível em: <http:// apsjournals.apsnet.org/doi/pdfplus/10.1094/PDIS-94-3-0284>. Acesso em: 28 dez. 2010. doi: 10.1094/PDIS-94-3-0284.

BERTOLDO, A.A. Desenvolvimento de metodologia para determinação dos componentes e especiação de polissulfetos em amostras de calda sulfocálcica. 2003. 87f. Dissertação (Mestrado em química) - Instituto de Química de São Carlos, Universidade de São Paulo, SP.

CASARIN, N.F.B. Calda sulfocálcica em pomares de citros: evolução da resistência em Brevipalpus phoenicis (Acari: Tenuipalpidae) e impacto sobre Iphiseiodes zuluagai (Acari: Phytoseiidae). 2010. 94f. Tese (Doutorado em Entomologia) - Escola Superior de Agricultura "Luiz de Queiroz", Universidade de São Paulo, Piracicaba, SP. Disponível em: $<$ http://www.teses.usp.br/teses/disponiveis/11/11146/tde20042010-101142/es.php>. Acesso em: 15 jan. 2010.

FERNANDES, M.C.A. Benefícios da utilização de defensivos alternativos no cultivo de hortaliças e fruteiras. In: HEIN, M. Resumo do $1^{\circ}$ encontro de processos de proteção de plantas: controle ecológico de pragas e doenças. Botucatu: Agroecológica, 2001. Cap.13, p.177-180.
MATUO, T. et al. Efeito de alguns adjuvantes da pulverização nas propriedades físicas do líquido. Summa Phytopathologica. Jaguariúna, v.15, p.163-173, 1989.

NAKANO, O. et al. Entomologia econômica. Piracicaba: Livroceres, 1981. 314p.

OLIVEIRA, M.L. de. et al. Influência do resíduo de calda sulfocálcica na eficiência do Vertimec $18 \mathrm{CE}$ no controle de Phyllocoptruta oleivora, em citros. In: CONGRESSO BRASILEIRO DE ENTOMOLOGIA, 19., 2002, Manaus, AM. Resumos... Manaus: Sociedade Entomológica do Brasil (SEB), 2002. p.252.

PATTARO, F.C. Calda sulfocálcica no agrossistema citrícola. 2003. 73f. Dissertação (Mestrado em Agronomia, Entomologia Agrícola) - Faculdade de Ciências Agrárias e Veterinárias, Universidade Estadual Paulista, SP.

PRATES, H.S. Caldas bordalesa, sulfocálcica e viçosa produtos alternativos na citricultura. 1999. Disponível em: <http://www.cati.sp.gov.br>. Online. Acesso em: 10. jun. 2010.

RODRIGUES, J.C.V. et al. Citrus leprosis virus vectored by Brevipalpus phoenicis (Acari: Tenuipalpidae) on citrus in Brasil. Experimental and Applied Acarology, Amsterdan, v.30, n.1, p.161-179, 2003. Disponível em: <http://www.springerlink.com/ content/m5n0x02410277q81/>. Acesso em: 28 dez. 2010. doi: 10.1023/B:APPA.0000006547.76802.6e. 